\title{
Augmented Reality Assembly Guidance Method Based on Situation Awareness
}

\author{
Xincan Zhao ${ }^{1+}$, Yupeng Sun ${ }^{1}$ \\ ${ }^{1}$ School of Information Engineering, Zhengzhou University, CO 450001, China
}

\begin{abstract}
In order to fulfill the maintenance and support task of adaptive Augmented Reality (AR) in complex situations such as environmental changes and product mobility in industrial scenarios, an augmented reality assembly guidance method based on situation awareness is proposed. Firstly, the digital twins corresponding to the physical model are constructed based on the digital twin technology to map the data and information of the entity in real-time. Secondly, the AR equipment is reconstructed to have the ability of deep environmental awareness in order to achieve accurate three-dimensional registration. Finally, situation awareness is realized by multi-sensor fusion, and the information guide is enhanced by fusion for the change of external environment and equipment status. The experimental results show that the augmented reality assembly guidance system based on the concept of digital twin can effectively perceive the external abnormal environment changes and the position and posture of components. According to the corresponding situation, adaptive trigger augmented reality information push, the real-time frame rate in the process of augmented reality guidance can reach 60 FPS.
\end{abstract}

Keywords: multi-sensor fusion, digital twins, augmented reality assembly induction, situational awareness

\section{Introduction}

Maintenance support is an important means to restore and maintain equipment performance. As a complex optical-mechanical system, the assembly process of equipment is designed with thousands of parts, fixtures, measuring tools and assembly operations. Augmented reality assembly instruction is augmented reality[1][2][3] is introduced into the virtual assembly system, through the real environment as the background, the assembly not only realizes the person and the real part of interaction, at the same time enhances the users perception of assembly instruction information[4][5], augmented reality assembly instruction can greatly improve the efficiency of assembly, improve the quality of security. However, in the complex environment, the traditional augmented reality assembly guidance could not perceive the state information of the assembly parts, and could not make corresponding measures for the changes in the external environment. Situational awareness is an environment-based, dynamic and holistic ability to perceive risks[6][7], which is based on information data and achieves the purpose of decision-making or action through acquisition, understanding, reality, and prediction. In this paper, the traditional augmented reality assembly guidance cannot accurately perceive the external environment and the state of components, and the traditional augmented virtual CAD (Computer-Aided Design) model information cannot be changed. This paper proposes an augmented reality technology based on the combination of situational awareness and digital twin technology of multi-sensor fusion. This method aims to carry out CAPP (Computer Aided Process Design) under the digital twin environment[8][9], plus multi-sensor fusion interaction algorithm to achieve the situational awareness of the device, and to implement adaptively triggered augmented reality based on changes in the environment and its state Guidance on assembly information.

+ Corresponding author. Tel.: + 15037128341 .

E-mail address: 704126774@qq.com 


\section{Capp Design Based on Digital Twin}

\subsection{Application of CAPP in Process Manufacturing}

Computer-Aided Process Planning (CAPP) is the application of information technology in the field of product manufacturing Process design. As one of the core contents of digital manufacturing technology, it is the bridge and link connecting CAD and CAM, as well as the important prior guiding knowledge for equipment maintenance [10]. In recent years, CAPP has made certain developments in the field of intelligence: for example, Kang et al.[11] used ontology technology to model process knowledge, established an ontology-based process decision expert system, and realized automatic selection and sequencing of processing methods for processing features; Huang Rui et al. [12] proposed the feature-level process design reuse of parts driven by 3D CAD model retrieval from the perspective of process reuse. However, at this stage, there are still two major problems in the application and development of CAPP. First, the traditional CAPP system is still a static machining process solution. That is after the design process ends, its corresponding CAD model is solidified, and it is impossible to achieve the actual disturbance Factors to give feedback; Secondly, a large amount of data existing in design and production cannot be fully utilized.

To sum up, process design, as a key link in the manufacturing of mechanical products, is inseparable from the decision-making, reasoning and optimization process of the whole product life cycle data, so how to manage and utilize the model information is particularly necessary.

\subsection{Design of 3D Process System For Digital Twins}

Aiming at the problems of traditional CAPP, this article intends to use digital twin technology as an improvement. On the basis of traditional CAPP, in order to meet the process design needs in the digital twin environment, this paper proposes the overall framework of a three-dimensional process design system in the digital twin environment to realize the external information of physical entities. The design process is shown in Fig. 1.

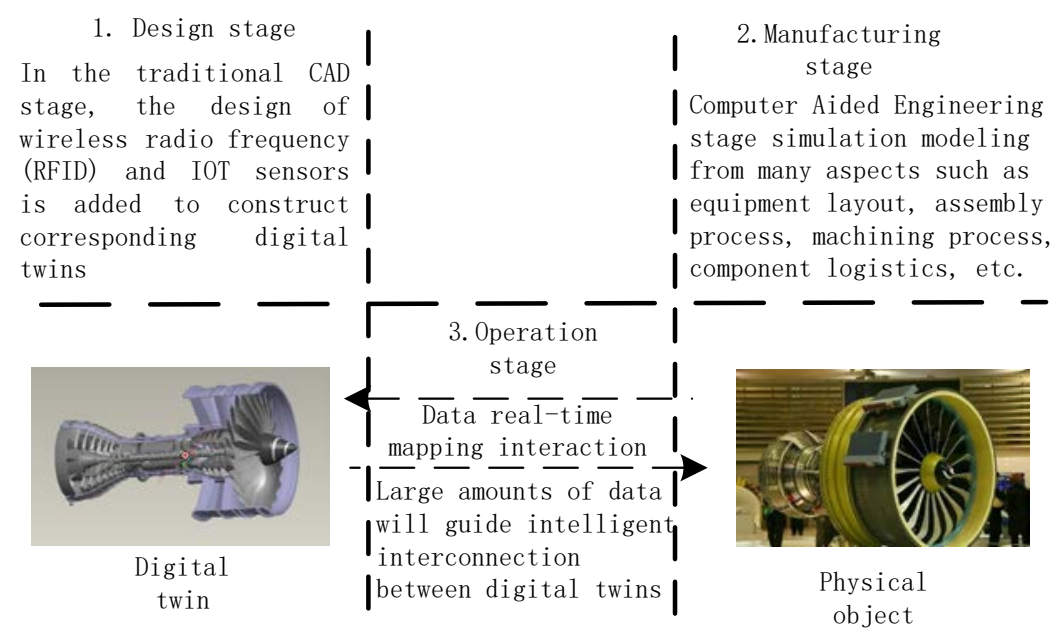

Fig. 1: CAPP design in digital twin environment

CAPP under the digital twin environment design innovates the digital twin which is different from the previous static model. Table 1 summarizes the similarities and differences between the digital twin and the traditional CAPP design.

Table. 1: Comparison of differences between digital dialysis organisms and traditional CAD models

\begin{tabular}{|l|l|l|}
\hline \multicolumn{1}{|c|}{ Innovation points } & \multicolumn{1}{c|}{ Subhead Product digital twin } & \multicolumn{1}{c|}{ Traditional CAPP design } \\
\hline Design method & Collaborative design of physical and virtual fusion & Designing in a virtual environment \\
\hline Driving method & Twin data-driven & Prior knowledge-driven \\
\hline Data management phase & $\begin{array}{l}\text { Product design, manufacturing, maintenance, and } \\
\text { other life cycle stages }\end{array}$ & Focus on the product design stage \\
\hline interactive mode & Twin data interaction & Offline interaction \\
\hline Ways of identifying & Real data simulation verification & $\begin{array}{l}\text { Trial production of small-batch } \\
\text { products }\end{array}$ \\
\hline
\end{tabular}




\section{Situational Awareness of Multi-sensor Fusion Algorithm}

\subsection{Multi-Sensor Fusion System Composition}

In this paper, the situational awareness of the system is realized through the embedded IOT sensors in the CAPP phase of digital twin guidance and multi-sensor fusion analysis and processing information. The multi-sensor fusion framework is shown in Fig. 2.

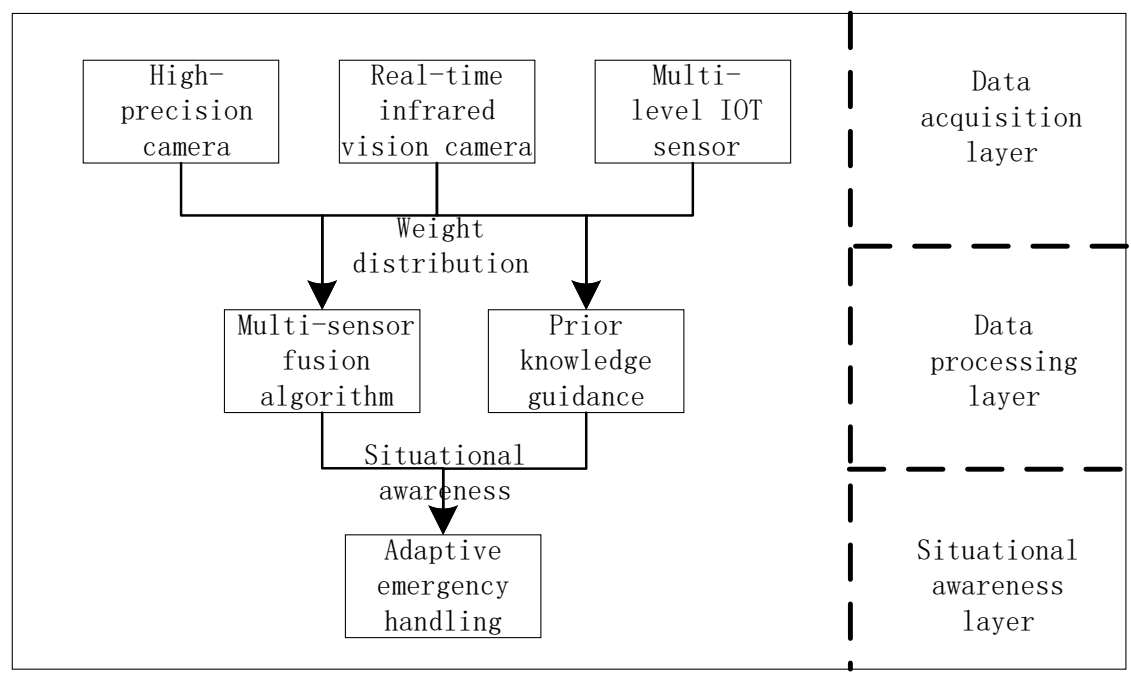

Fig. 2: Multi-sensor fusion for situational awareness

This article first uses data twinning technology as a guide at the equipment component stage, and completes data acquisition through pre-embedded IoT temperature and humidity sensors, Microsoft HoloLens augmented reality headset and Intel ${ }^{\circledR}$ RealSense. Then, the improved point cloud KLD particle filter algorithm is adopted for object 6-DOF perception and tracking. Finally, the ability of situational awareness is achieved by combining temperature and humidity sensors and prior knowledge. The scene recognition diagram is shown in Fig. 3.

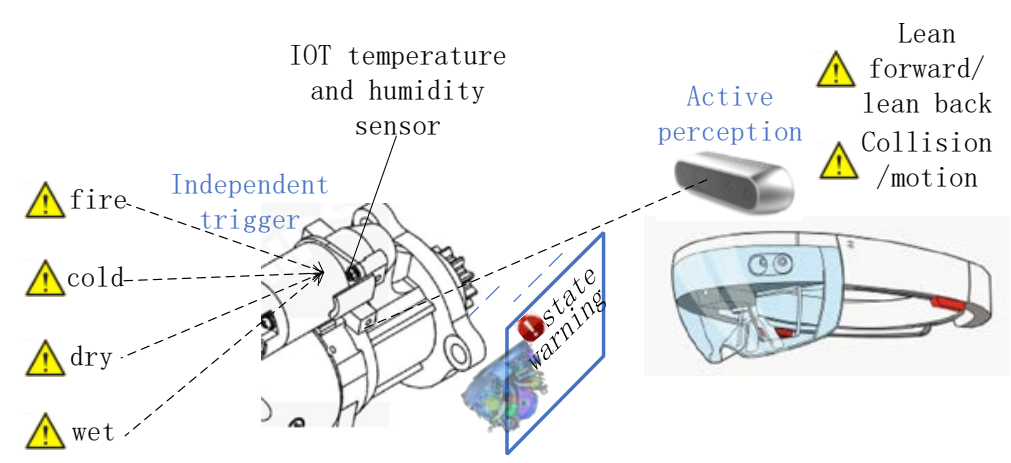

Fig. 3: Situational awareness diagram

\section{Situational awareness guided by prior knowledge}

\subsection{Active Perception Based on Visual Data}

As traditional MEMS acceleration sensor (such as gyro, accelerometer) can only get the object position information and unable to get space, the real goal is portable high-precision depth camera Intel RealSense sensors components as external visual information in the space of real-time 6-DOF and real-time six degrees of freedom of the acceleration value.

This paper proposes to use an improved KLD particle filter algorithm to obtain the real-time 6-DOF attitude of the target, where KLD is Kullback-Leibler divergence, also called relative entropy. It can describe the difference in distribution between the two probabilities. It mainly measures the certainty of the posterior 
probability distribution in the particle filter algorithm. The method of dividing into different small regions can be used to discretize the posterior probability density distribution of particles. Suppose there is a vector $X=\left(X_{1}, \cdots, X_{k}\right)$ Represented as the number of sampled particles in some different regions, then obey the polynomial distribution $X=\operatorname{Multinomial}_{k}(n, p)$ among them $p=p_{1}, \cdots, p_{k}$, Represents the probability of sampling into each region. According to statistical principles, The maximum likelihood estimate of $p$ is , The likelihood statistic for test $p$ is $\hat{p}=n^{-1} X$ :

$$
\log \lambda_{n}=\sum_{j=1}^{k} X_{j} \log \left(\frac{\hat{p}_{j}}{p_{j}}\right)=n \sum_{j=1}^{k} \hat{p}_{j} \log \left(\frac{\hat{p}_{j}}{p_{j}}\right)
$$

It $p$ is a true posterior distribution, when $n \rightarrow \infty$, Likelihood ratio converges to $\chi^{2}$ distribution:

$$
2 \log \lambda_{n} \rightarrow d \chi_{k-1}^{2}
$$

Then the cumulative sum of the right term of Eq. (1) is the relative entropy of the maximum similar then posterior distribution and the true posterior distribution. Then the relationship between the probability that the Kullback-Liebler distance is less than $\varepsilon$ and the number $n$ of samples drawn from the posterior density is:

$$
\begin{aligned}
& p_{p}(K(\hat{p}, p) \leq \varepsilon)=p_{p}(2 n K(\hat{p}, p) \leq 2 n \varepsilon) \\
& =p\left(\chi_{k-1}^{2} \leq 2 n \varepsilon\right)
\end{aligned}
$$

Since the $\chi^{2}$ distribution quantile is:

$$
p\left(\chi_{k-1}^{2} \leq \chi_{k-1,1-\delta}^{2}\right)=1-\delta
$$

Then, when $n$ satisfies $2 n \varepsilon=\chi_{k-1,1-\delta}^{2}$, that is, $n=\frac{\chi_{k-1,1-\delta}^{2}}{2 \varepsilon}$, then

$$
p_{\underline{p}}(K(\underline{\hat{p}}, p) \leq \varepsilon)=1-\delta
$$

Then, Wilson-Hilferty transformation is adopted to simplify the operation:

$$
n=\frac{\chi_{k-1,1-\delta}^{2}}{2 \varepsilon}=\frac{k-1}{2 \varepsilon}\left\{1-\frac{2}{9(k-1)}+\sqrt{\frac{2}{9(k-1)}} z_{1-\delta}\right\}^{3}
$$

Where $z_{1-\delta}$ represents the upper bound of the quantile of $1-\delta$ in the standard normal distribution, The KL distance of the number of particles required by the t-step filter is inversely proportional to $\varepsilon$ and proportional to the threshold value $\Delta$ of the small region.

Based on adaptive particle filter algorithm based on prior knowledge to guide track of improved, with the first frame point cloud into early recognition and registration of 6-DOF as tracking template, will initialize the particle distribution is based on the CAD model of the prior knowledge , thereby greatly reducing the tracking process of initialization time so as to improve the accuracy of the initial tracking, algorithm diagram as shown in Fig. 4.

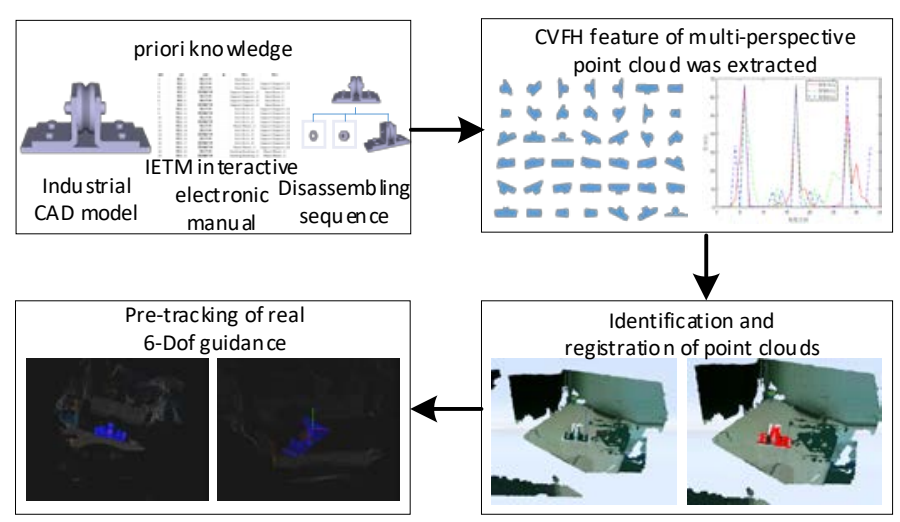

Fig. 4: Particle filter tracking algorithm guided by prior knowledge 


\subsection{Multi-Sensor Data Fusion Processing}

Multi-sensor collaborative operation has significant advantages in fault tolerance, complementarity, realtime and other aspects. However, since the state transfer threshold of different components is different, the actual measurement alarm threshold is introduced as the prior reference value. Taking a single-input singleoutput sensor as an example, taking a certain sensor as an example, assuming that the input quantity is $\mathrm{x}(\mathrm{t})$, the noise is $\mathrm{N}(\mathrm{t})$, and the output quantity is represented by $\mathrm{y}(\mathrm{t})$, then the differential equation can represent the dynamic characteristics of the sensor[13]:

$$
\begin{aligned}
& a_{n} y^{n}(t)+a_{n-1} y^{n-1}(t)+\cdots+a_{1} y^{1}(t)+a_{0} y^{0}(t)=b_{m} x^{m}(t)+b_{m-1} x^{m-1}(t)+\cdots+b_{1} x^{1}(t)+b_{0} x^{0}(t) \\
& +c_{p} N^{p}(t)+c_{p-1} x^{p-1} N(t)+\cdots+c_{1} N(t)+c_{0}
\end{aligned}
$$

Among them $a_{0}, a_{1} \cdots a_{n}, b_{0}, b_{1}, \cdots b_{m}, c_{0}, c_{1} \cdots c_{p}$ are the coefficient. When the threshold of a single sensor is selected, a critical threshold with $T_{a}^{1}$ slightly smaller danger threshold is taken as the trigger threshold, when $T_{a}^{1}<T_{a}^{0}$ :

$$
\begin{aligned}
& T_{a}^{1}=\left\{\begin{array}{l}
\bar{X}+3 X_{s}, T_{a}^{1}<T_{a}^{0} \\
0.9 T_{i}, T_{a}^{1}>T_{a}^{0}
\end{array}\right. \\
& \omega_{i}=\left\{\begin{array}{l}
0, v_{t}<T_{a}^{1} \\
1, v_{t}>T_{a}^{1}
\end{array}\right.
\end{aligned}
$$

Where $\omega_{i}$ is the weight of a single sensor, $v_{t}$ is the actual measured value, $\bar{X}$ is the historical average value of the sensor, and $X_{s}$ is the standard deviation of the historical data collected by the sensor[14]. Considering that the numerical weight of different sensors will be affected by dimension, that is, the weight scale is different, this paper intends to use Markov distance to adjust the weight $\mathbf{a}, \mathbf{b}$ are members of two different vectors:

$$
D_{M}(\mathbf{a}, \mathbf{b})=\sqrt{(\mathbf{a}-\mathbf{b})^{T} \sum^{-1}(\mathbf{a}-\mathbf{b})}
$$

Where $\sum^{-1}$ represents the covariance matrix of the vector, then the dangerous state score is:

$$
R=D_{M}\left(\left(T_{a}, 0\right), \mathbf{0}\right) \times D_{M}\left(\left(T_{b}, 0\right), \mathbf{0}\right) \times D_{M}\left(\left(T_{c}, 0\right), \mathbf{0}\right)
$$

Where $T_{a, b}, c$ is the threshold value of different sensors [15] Its flow chart is shown in Fig. 5.

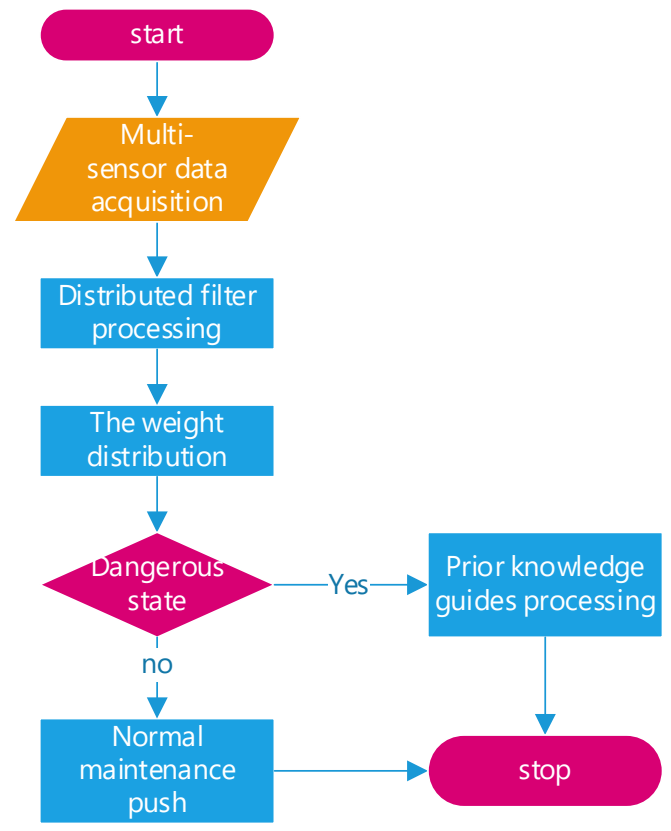

Fig. 5: Multi-sensor fusion data processing flow

\subsection{Situational Awareness and State Decision Based on Prior Knowledge Guidance}

After multi-sensor fusion, the system has acquired the ability to perceive the external environment and its own state, so the decision making under different environments and states becomes particularly important. 
The perception and prediction in different states can be regarded as a series of discontinuous states, so the finite state automata can be used to establish a discrete mathematical model to represent the behavior of migration between these states. The decision finite state machine can be expressed as: $t_{0} \in T$ is the initial state, and $F \subseteq T$ is the final state. Where $T$ is the set of all states in the state machine; $E$ is the set of acceptable inputs between states (that is, the trigger conditions for state transition); $\delta$ is the state transfer function, expressed as $\delta: T \times E \rightarrow T$; The finite-state automaton decision diagram is shown in Fig. 6 .

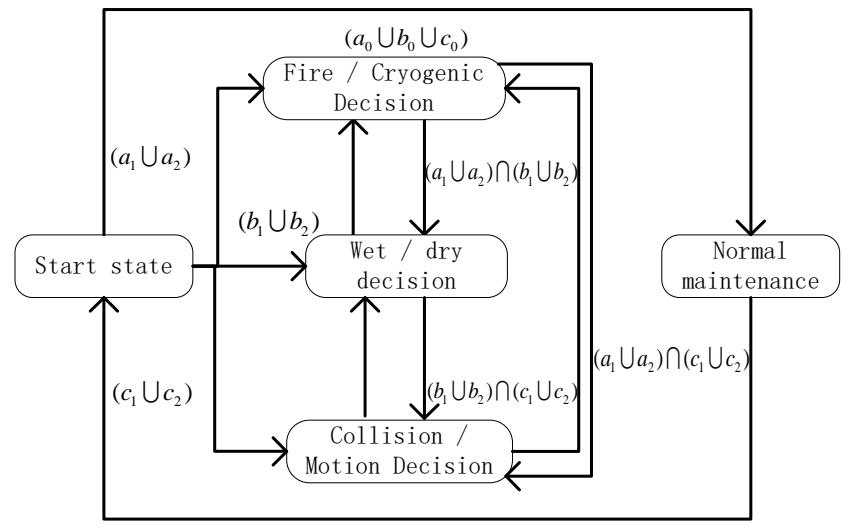

Fig. 6: Finite-state automaton decision diagram

$a_{0} 、 b_{0} 、 c_{0}$ represents the historical measurement average value of the sensor, $a_{1} 、 a_{2}$ represents the fire and low-temperature early warning threshold, $b_{1} 、 b_{2}$ represents the wet and dry early warning threshold, and $c_{1} 、 c_{2}$ represents the collision motion early warning threshold.

\section{System Implementation}

\subsection{Experimental System Setup}

This article incorporates the concept of digital twins and considers the embedded sensor during the component-aided design stage. The comparison of the CAD model design schematic with the real production component is shown in Fig. 7.
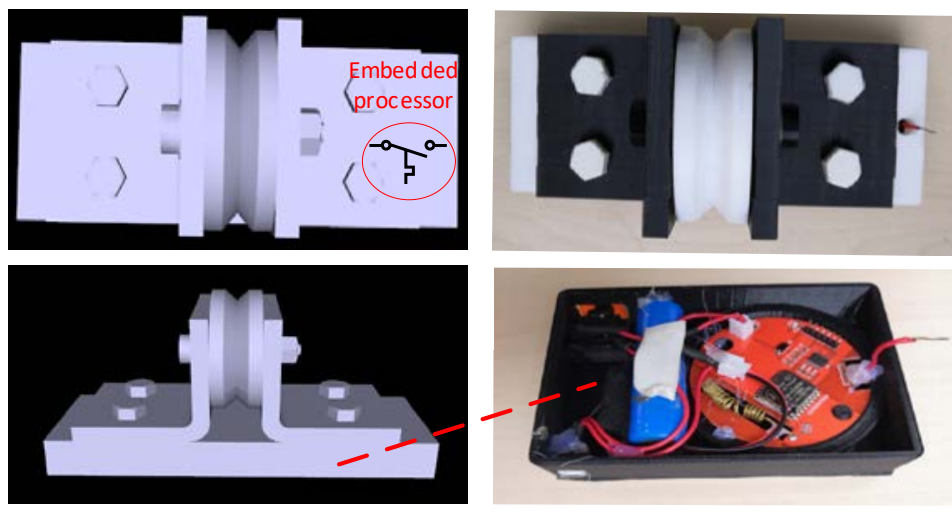

Digital twin

Physical entity

Fig. 7: Model design incorporating digital twin concepts

The IoT low-power sensor has a built-in lithium battery, and its sensing capability is parameterized as follows:

Table. 2: parameters of embedded sensors

\begin{tabular}{|l|l|l|}
\hline $\begin{array}{l}\text { Measurem } \\
\text { ents }\end{array}$ & \multicolumn{1}{|c|}{ Measuring range } & error \\
\hline $\begin{array}{l}\text { Temperatu } \\
\text { re }\end{array}$ & $-20^{\circ} \mathrm{C} \sim 60^{\circ} \mathrm{C}$ & $\pm 0.5^{\circ} \mathrm{C}$ \\
\hline Humidity & $0 \% \sim \sim 100 \%$ & $\pm 2 \%$ \\
\hline $\begin{array}{l}\text { Air } \\
\text { pressure }\end{array}$ & $300 \mathrm{hpa} \sim 1100 \mathrm{hpa}$ & $\pm 50 \mathrm{hpa}$ \\
\hline
\end{tabular}


This article uses Microsoft HoloLens augmented reality headset as the basic hardware of the experiment. In order to realize accurate real-time six-DOF sensing of components, Intel RealSense is used as an external high-precision 3D vision sensor. Its design and physical matching are shown in Fig. 8.
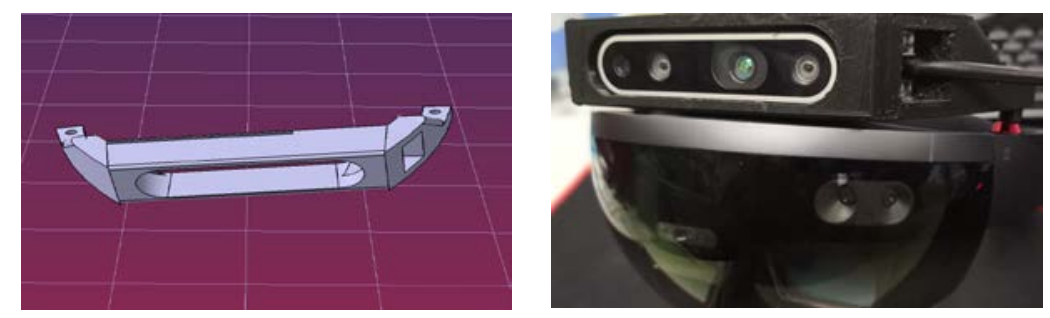

Fig. 8: Model design incorporating digital twin concept

The complete augmented reality maintenance in-duction system also includes a mini-host for real-time information processing and an independently powered portable power supply, as shown in Fig. 9. The minihost is responsible for processing all sensor data and status information decision-making, and communicating with the HoloLens helmet in real-time via UDP/TCP for 6-DOF and warning status.

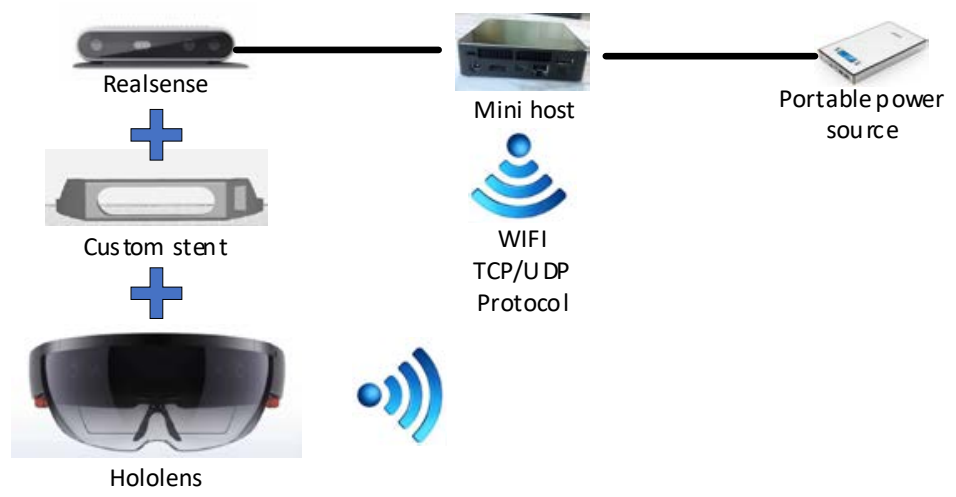

Fig. 9: Structure diagram of AR maintenance induction system

In different stages of the visual perception of the experiment, result is shown in Fig. 10 and figure a for visual perception capture process, figure b said prior knowledge to guide the parts identification, figure c said after identification of $3 \mathrm{~d}$ virtual prototype model and entity model, figure $\mathrm{d}$ said in part moves for the capture of the real-time status, redpoint for tracking particles in the component state, blue model for virtual assembly model. Visual information processing uses OSG(OpenSceneGraph) as the graphics rendering and processing engine, combined with the PCL point cloud library for 3D point cloud recognition and registration, and then fuses the prior model with the real thing.
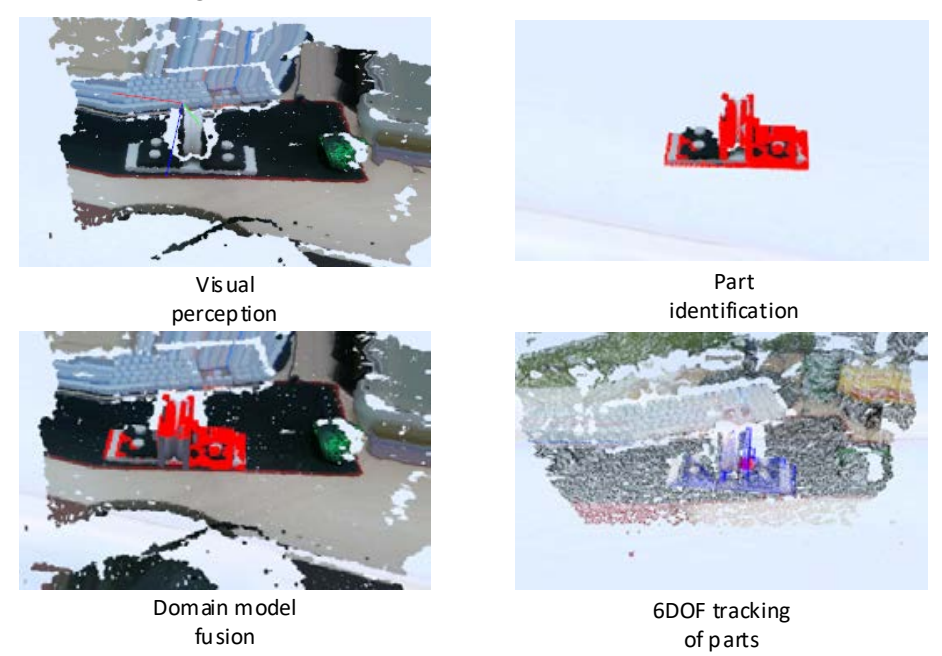

Fig. 10: Effects of visual perception at various stages 
Experiments show that the part identification is accurate and the model registration effect is obvious, which can effectively guide practitioners to understand the physical state information, and the real-time assembly instruction in HoloLens can reach the frame rate of 60fps.

\subsection{Augmented Reality Induction Under Situational Awareness}

At the beginning of the task, the real-time position of the part in space was obtained through visual perception, accurate 3D assembly guidance information was superimposed according to the state of the part, and complete process animation was pushed forward in front of the field of vision, so as to guide the staff to complete the assembly induction task according to the state of the part assembly. The device's own state perception and its augmented reality assembly guidance effect are shown in Fig. 11.

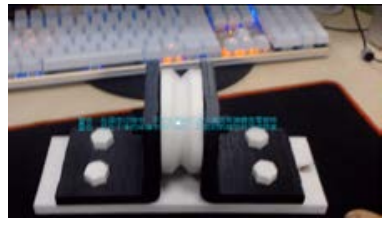

a. Maintenance support tasks

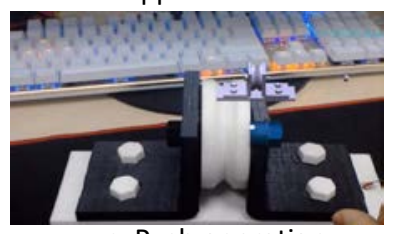

c. Push operation steps

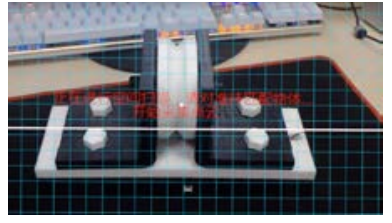

b. 3D scene perception

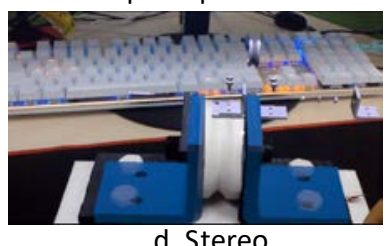

d. Stereo

information fusion

Fig. 11: Self-awareness and enhanced information guidance

In the fault maintenance detection task, the component senses the external temperature, humidity, and air pressure in real-time through its own sensor, determines the fault status based on the trigger threshold, sends a fault warning to the system when the external temperature is abnormal, and detects different fault codes based on the real-time status, The corresponding guidance information and operation process are holographically projected onto the physical entity, and the maintenance staff completes the troubleshooting according to the troubleshooting content until the equipment detection environment returns to normal. The situational awareness and warning effects of simulated external conditions are shown in Fig. 12.

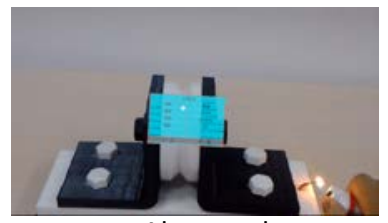

a. Abnormal temperature outside

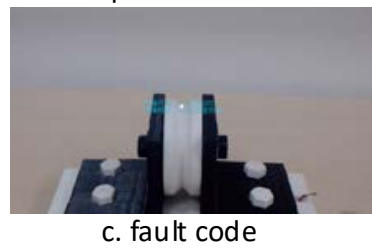

push

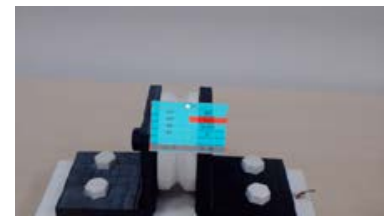

b. System situational awareness

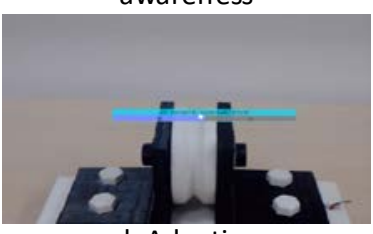

d. Adaptive

Information Decision

Fig. 12: Situational Awareness and Information Decision

\section{Conclusion}

Aiming at problems such as the weak perception of changes in the external environment and its own state in augmented reality applications, and augmented reality situation awareness system that incorporates the concept of digital twins is proposed. Through multi-sensor fusion and digital twin information interaction, abnormal changes in the external environment and changes in its own state. The perception and decisionmaking have laid a certain foundation for the maintenance support of augmented reality in complex scenarios. 
In the future, we can continue to improve the robustness of the system under complex scene changes to make it more complete.

\section{Acknowledgment}

The work in this paper was supported by the National Natural Science Foundation of China (\# U1433106, \#61603344) and the Aviation Science Foundation of China(2018ZC41002).

\section{References}

[1] Caudell T, Mizell D. Augmented reality: an application of heads-up display technology to manual manufacturing processes[C].//Proceedings of the 25th Hawaii International Conference on System Sciences. Los Alamitos: IEEE Computer Society Press, 1992: 659-669.

[2] Gao Xiang, Hui Chen, Wei Pan,Zhi geng. A Survey on Mobile Augmented Reality Visualization [J]. Journal of Computer-Aided Design and Computer Graphics,2018,30(01):1-8.

[3] Ren D, Goldschwendt T, Chang Y, et al. Evaluating wide-field-of-view augmented reality with mixed reality simulation[C].//Proceedings of the IEEE Virtual Reality. Los Alamitos:IEEE Computer Society Press, 2016: 93102.

[4] Research on Auxiliary maintenance system of aircraft engine based on augmented reality [J].Science and Technology Innovation Herald,2019,16(06):16-18.

[5] XU Yi-da. Research on fault diagnosis and maintenance technology of civil aircraft assisted by augmented reality[J]. Science \& Technology Vision,2018(17):7-8.

[6] Qu Yang.Marine Environmental data management and Military Applications[J]. Electronic Technology \& Software Engineering,2019(14):171-172.

[7] Cao guoxi.Research on evaluation system for UAV operator situation awarness[D].Beijing University of Posts and telecommunications,2019.

[8] LI kai, Qiao hao,Gong Mengyao Wang Xiaolei. Digital Warship and its Application Exploration Based on Digital Twin Technology[J]. Ship \& Boat,2018,29(06):101-108.

[9] Tao Fei, Cheng Ying, Cheng Jiangfeng, Zhang Meng, Xu Wenjun, Qi Qinglin. Theories and technologies for cyber-physical fusion in digital twin shop-floor[J]. Computer Integrated Manufacturing Systems,2017,23(08):1603-1611.

[10] YuYong, Hu Deyu, Dai Sheng, Zhao Gang. Study on Application of Digital Twin in Process Planning[J]. Aeronautical Manufacturing Technology,2018,61(18):26-33.

[11] Kang M, Kim G , Lee $T$, et al. Selection and sequencing of machining processes for prismatic parts using process ontology model[J].International Journal of Precision Engineering and Manufacturing, 2016, 17(3): 387-394.

[12] Huang Rui, Zhang Shengheng, Bai Xiaoliang, Zhang Congcong, Zhang Ximao. 3D CAD Model Retrieval Driven Rapid NC Programming Method for NC Process Reuse[J]. Journal of Mechanical Engineering,2014,50(03):191198.

[13] Zhao Yuesheng.MEMS Bionic Individual Based on Prior Knowledge, Real-time Situation Awareness and Interactive Learning[D].Tsinghua University,2013.

[14] Hu Zhenyu. Analysis of Photoelectric Multi-sensor Information Fusion Technology Based on Target Recognition[J]. China Computer \& Communication,2019(11):139-140.

[15] Sun Xiaoli. Application of multisensor information fusion in robotics[J]. Wireless Internet Technology,2018,15(02):130-131. 